\title{
Evaluación formativa a procesos creativos en programas de artes visuales nivel superior México: acercamiento mediante estudio de caso.
}

Training process evaluation in visual arts programs superior level México: approach through case study.

\author{
Víctor Guillén García \\ Irma Flores Alanís 1 \\ Universidad Autónoma de Nuevo León, México
}

\section{Resumen}

La educación para la formación de artistas (visuales, escénicos, arquitectos, músicos y áreas afines) fuera de las academias del siglo XIX, es un proceso que a partir de la segunda mitad del siglo XX ha sido insertado en el currículo y programas de estudio de nivel superior, campo al cual se han agregado nuevos cuestionamientos e inquietudes respecto a las estrategias de enseñanza idóneas, específicamente para el ámbito de referencia de la presente tesis: la evaluación de la creatividad realizada por el docente. Se partirá del supuesto que dicha valoración encuentra sus pilares dentro de los procesos formativos involucrados en la investigación, creación y producción artística de los estudiantes. En el planteamiento anterior se presenta una de las complejidades para la evaluación; objetivar con un sentido crítico, dentro de los talleres teórico-prácticos en programas académicos de licenciaturas en artes, no solo productos y resultados, sino también procesos, prácticas y significados a cuestiones creativas que, por su constante evolución y naturaleza, pueden tornarse subjetivas, lo cual ha desembocado en un debate de dimensiones internacionales (Borgdorff, 2005; Díaz Alcaide, 2011; Storr, 2009). El artículo a desarrollar es sobre la dinámica y alcances de la evaluación formativa que contemple el proceso creativo involucrado en el área de talleres en artes visuales del nivel superior en México, fundamentada en la exploración y análisis de estudios previos sobre la educación y evaluación en disciplinas artísticas, para analizar información que aporte ideas que clarifiquen aspectos susceptibles a evaluarse observando procesos de Investigación y Creación artística (IC) nacidos en programas escolarizados.

Palabras clave: evaluación formativa; educación artística; artes visuales.

\begin{abstract}
:
Education for the training of artists in disciplines such as the visual arts, theatre, architecture, music and related areas outside of the academies of the 19th century, is an activity that only as recently as the second half of the 20th century has been inserted into the academic curriculum of higher education. Since that point there have been questions, debates and concerns related to the strategies of said education corresponding to assessments made by educators involved in creative processes and artistic production. The challenge exists to develop an objective, critical system of evaluation related to processes, practices and creative issues in art education that by the constant evolution of the arts is often considered subjective, which has resulted in a debate of international dimensions (Borgdorff, 2005; Díaz Alcaide, 2011; Storr, 2009). The article to be developed is about the dynamics and scope of the formative evaluation that contemplates the creative process involved in the area of workshops in visual arts of the highest level in Mexico.
\end{abstract}

Keywords: formative evaluation, art education, fine arts.

\footnotetext{
${ }^{1}$ alejandro4074@outlook.es; irma.floresal@uanl.edu.mx
} 


\section{Introducción}

La investigación en curso tiene como objeto de estudio la evaluación formativa en talleres del área de artes visuales del nivel superior y cómo se realiza; si se considera el análisis de procesos creativos implícitos en la investigación y producción derivados como unidad de aprendizaje y evaluación en áreas teórico-prácticas de licenciatura; qué aplicación se da a la información obtenida, así como la pertinencia en los grados de ponderación e indicadores de la misma. La relevancia de tal enfoque radica en considerar que, en la medida que se contemple dentro de la evaluación, a dicha unidad de proceso (IC) como parte integral del aprendizaje en artes, éste puede verse beneficiado gracias a una visión con mayor amplitud y alcances, traducidos en un tipo de evaluación formativa.

Para analizar los procesos de IC primero es necesario conocer las partes que les son subyacentes: el planteamiento de la actividad a realizar, la investigación formal o intrínseca, desarrollo y sistematización del trabajo, conceptualización, creación y evidencias finales que demuestren la concreción de los objetivos descritos en el programa de la asignatura de estudio en el taller en cuestión (pintura, escultura, dibujo, fotografía, instalación, etcétera) de artes visuales.

Michaud (2003) escribe respecto la existencia de dos grandes grupos donde se mueve la manifestación artística en el siglo XXI; uno donde se rinde culto, casi de adoración a la belleza prefabricada, el triunfo de la estética profusa, a la experiencia estética pasajera y sin soportes, casi gaseosa; y en otro extremo, pero con el mismo resultado, la saturación y reproductibilidad seriada del objeto artístico, la transformación de los museos y galerías en supermercados del arte. Con el seguimiento de dichas líneas, la evaluación del objeto artístico, puede sesgarse hacia la estandarización de tendencias esteticistas para consumo (real o abstracto) de los espectadores (Spentsas 218).

La evaluación realizada de tal forma, o en el caso presente, del objeto artístico, se puede sesgar a los gustos o experiencias previas del espectador como consumidor (Romero 2011), o a la estandarización de tendencias esteticistas para consumo masivo. En el caso específico del sistema educativo, el nivel superior, dicha responsabilidad recae en primera línea en el profesor de asignatura, con el compromiso que se le adjudica desde los parámetros establecidos por el programa educativo, planes de estudio y políticas institucionales, en conjunción con el criterio, experiencia y gustos personales del profesor responsable de la evaluación.

El problema planteado nace del interés basado en observaciones y verificación respecto de cuáles son los indicadores que se consideran relevantes al evaluar la producción artística de los estudiantes en el nivel superior, los que se adjudican al dominio de habilidades de oficio (técnica), a los valores estéticos o al proceso y apropiación de la experiencia creativa del alumno.

Los juicios estéticos corresponden a contextos y realidades matizadas por la cultura y el conocimiento del grupo social donde la misma se realiza, por tanto, la complejidad puede tornarse mayor.

\section{Antecedentes}


La historia de la educación en artes visuales está ligada con la historia del arte y con la historia de la educación en general, para no caer en historicismos, se tomará como punto de partida el siglo XX, ya que es a principios de dicho siglo, en pleno debate sobre la modernidad (Efland D., Freedman, \& Stuhr, 2003), que las corrientes artísticas abandonan el ideal esteticista y dan paso a los movimientos conocidos como "las vanguardias" diferenciando dos ramas estéticas, la del formalismo y el expresionismo, lo anterior como parte de las tendencias a cuestionar el racionalismo vigente en los ámbitos sociales y culturales de la época postindustrial y de entreguerras. En la tabla 1 se presentan de manera cronológica los antecedentes históricos relevantes a la conformación de los modelos educativos y de evaluación en artes vigentes en la actualidad de México.

Tabla 1

Referentes históricos: educación y evaluación en artes. Elaboración propia

\begin{tabular}{|c|c|c|c|}
\hline Referente & Lugar & Período & Características \\
\hline $\begin{array}{l}\text { Escuela del } \\
\text { Bauhaus }\end{array}$ & $\begin{array}{l}\text { Weimar, } \\
\text { Alemania }\end{array}$ & $\begin{array}{l}1919- \\
1933\end{array}$ & $\begin{array}{l}\text { Promueve el trabajo desde diferentes disciplinas } \\
\text { artísticas. Se fusionan conocimientos de } \\
\text { artesanos con el de diseñadores, arquitectos, } \\
\text { pintores, escultores, fotógrafos y otros } \\
\text { profesionales de las artes para la enseñanza y la } \\
\text { práctica artística, en un movimiento frente a la } \\
\text { crisis del pensamiento racional y técnico de la } \\
\text { época. }\end{array}$ \\
\hline $\begin{array}{l}\text { Black } \\
\text { Mountain } \\
\text { College }\end{array}$ & $\begin{array}{ll}\text { Carolina } & \text { del } \\
\text { Norte, } & \\
\text { Estados } & \\
\text { Unidos } & \text { de } \\
\text { Amércia } & \end{array}$ & $\begin{array}{l}1933- \\
1957\end{array}$ & $\begin{array}{l}\text { Establecimiento experimental, dedicado a la } \\
\text { enseñanza de las artes. Educación } \\
\text { interdisciplinar, definida como educación } \\
\text { progresiva, en palabras de su fundador John Rice, } \\
\text { sus procedimientos enfatizan en enseñar método } \\
\text { y no contenido, dando mayor relevancia a los } \\
\text { procesos y no a los resultados (Erickson, 2016). } \\
\text { No se otorgan títulos oficiales para la obtención } \\
\text { de grados. }\end{array}$ \\
\hline $\begin{array}{l}\text { Joseph } \\
\text { Beuys, } \\
\text { Academia de } \\
\text { Bellas Artes } \\
\text { de } \\
\text { Düsseldorf }\end{array}$ & $\begin{array}{l}\text { Düsseldorf, } \\
\text { Alemania }\end{array}$ & $\begin{array}{l}1961- \\
1972\end{array}$ & $\begin{array}{l}\text { Educar en las artes de manera democrática e } \\
\text { integral, estimulando la reflexión y la creatividad, } \\
\text { más allá del perfeccionamiento de técnicas y } \\
\text { conceptos. Todo hombre, un artista. }\end{array}$ \\
\hline $\begin{array}{l}\text { Universidad } \\
\text { Libre } \\
\text { Internacional. } \\
\text { Joseph Beuy, } \\
\text { fundador. }\end{array}$ & Sin sede & $\begin{array}{l}1973- \\
1988\end{array}$ & $\begin{array}{l}\text { Derecho primordial a la formación, el apoyo } \\
\text { económico de los estudiantes para financiar la } \\
\text { universidad desde la base de acuerdo con la } \\
\text { voluntad de formación para: alimentos, ropa, } \\
\text { vivienda; retribución del profesorado; } \\
\text { mantenimiento y ampliación de las instalaciones } \\
\text { universitarias; equiparar los exámenes libres con } \\
\text { los exámenes de Estado. }\end{array}$ \\
\hline
\end{tabular}


En el caso de México, el estudio de las artes visuales en sistemas universitarios se integra a finales de la década de 1970, atendiendo a la visión sobre cómo la educación requeriría ser integral y abarcar todas las ramas de conocimiento, tanto científico como humanista, incorporando las escuelas y academias en artes a la oferta de licenciaturas, adecuándolas para la profesionalización de dicho campo, pero manteniendo la responsabilidad docente a cargo de los artistas expertos en las disciplinas artísticas tradicionales (pintura, escultura y grabado). El modelo disciplinar aplicado en las artes, presentó oposición por parte de la comunidad artística del país, que da surgimiento a una corriente conocida como "la nueva generación" como menciona Rodrigues Widholm que se plantea como parte de su ideología, mantener independientes a la educación académica y la práctica artística, por considerar a la primera como mensajera de parámetros institucionales que pudieran ser opuestos a la libertad creativa, obedeciendo a líneas específicas relacionadas con un mercado del arte.

Es a finales de la década de 1980 surgen espacios alternativos a la Escuela Nacional de Artes Plásticas (ENAP) o a la Escuela Nacional de Escultura, Pintura y Grabado "La Esmeralda"; para manifestar un acto de rebeldía, promoviendo exposiciones, análisis y nuevas visiones para entender, evaluar y despojar al arte de su sentido romántico e historicista; Temístocles 44, Foro de Arte Contemporáneo, La Panadería y SOMA, entre otros.

La importancia para mencionar dichos momentos históricos con la evaluación en talleres de artes visuales, radica en las propuestas y descontento de una generación al final del siglo XX, respecto al seguimiento mediante sistemas disciplinares, aplicados con pocas adecuaciones, para otorgar validez a la producción académica artística, sin tomar en cuenta la necesidad de entenderla desde una perspectiva focalizada en el entorno mexicano, para integrarse en términos mundiales, pero con una personalidad definida.

\section{Justificación}

Existe la necesidad de que todo proceso, objeto de evaluación, debe ir más allá del ejercicio de control y la asignación de calificaciones; cambiando el discurso de la misma para que se convierta en el producto de la reflexión y el entendimiento apoyados en acuerdos verbales entre los actores involucrados: el responsable de la evaluación (profesor) y los sujetos (estudiantes), ya que el proceso de evaluación es también un proceso de enseñanza y aprendizaje por la experiencia que nace en dicha interacción.

La evaluación en talleres de artes visuales, respecto de las evidencias del aprendizaje, debe considerar tanto los conocimientos adquiridos (teóricos); las cualidades (conceptuales, discursivas, analíticas, relaciones históricas y personales, motivacionales) y las capacidades (técnicas, de ordenamiento, limpieza) presentes en la investigación, creación y producción, para partir del entendimiento de la correspondencia a un trayecto indivisible, ya que, si bien pueden corresponder a objetivos y momentos diferentes, juntos son el reflejo de un desarrollo tanto cognitivo como cognoscitivo, que se acerca al desarrollo de un objeto o concepto de estudio, dentro de un contexto específico, estimulando nuevos conocimientos y nuevas relaciones entre las partes que le son subyacentes, por lo que en conjunto se manifiestan como evidencias de los procesos teóricos, creativos y de habilidades técnicas. 


\section{Objetivos}

Los objetivos del trabajo de investigación de la tesis serán:

- Diseñar un modelo para evaluar procesos creativos en talleres de artes visuales del nivel superior en México

Para conseguirlo ha de ser necesario un plan de trabajo que incluya:

- Diagnosticar el tipo evaluación realizada a procesos inherentes a la creatividad, y su relación con la IC en talleres de artes visuales del nivel superior en México.

- Identificar interrelaciones entre el perfil del docente, el perfil del estudiante y el contexto institucional, para la evaluación en talleres de artes visuales.

- Examinar y contrastar las diferentes posturas y criterios de evaluación llevados a la práctica en las artes visuales.

\section{Pregunta de investigación}

Partiendo de la afirmación de que los procesos creativos son inmanentes a la investigación y producción artística, y estos son susceptibles a ser evaluados ¿Cómo evaluar el proceso creativo, desde el ámbito formativo, en los talleres de artes visuales del nivel superior? Para dar respuesta, se procederá a seleccionar el método de investigación que se revele como el más adecuado, que en el caso presente, por las características involucradas desde las humanidades, será la Teoría Fundamentada (TF).

\section{Supuestos}

- La evaluación formativa es adecuada para valorar el proceso creativo, expresado en términos de cualidad, calidad y cantidad. Ya que en esencia, tal tipo de evaluación en su desarrollo se retroalimenta, se redirecciona, y se recrea continuamente, lo cual le liga al proceso de la IC para su valoración.

- Las actividades de investigación en y desde las artes, están estrechamente ligadas al proceso creativo de producción, lo que abre alternativas para su medición y evaluación, desde un enfoque tanto cualitativo como cuantitativo.

\section{Nociones teórico-operativas \\ 1.1. Evaluación formativa}

Una evaluación será formativa en la medida que las evidencias de la los aciertos y errores de los estudiantes sirven para registro e interpretaciones que promuevan nuevas estrategias y den como resultado un mayor y mejor aprendizaje, es decir, la evaluación se construye a sí mísma constantemente durante el curso y no es solo una herramienta de medición al finalizarlo.

\subsection{Arte}

Se tomará la referencia de Tatarkiewicz quien agrupa las manifestaciones u obras de arte según la intención (configurar la realidad o la necesidad de expresión); el efecto (experiencias estéticas, deleite, sorpresa o producir un choque); los productos y su relación con la realidad (reproducciones de la realidad o abstracciones); el valor (belleza, gracia delicadeza, sublimidad, experiencias estéticas). "El arte es una actividad humana consciente capaz de reproducir cosas, construir formas, o expresar una experiencia, si el producto de esta reproducción, construcción, o expresión puede deleitar, emocionar o producir un choque" (67)

\subsection{Creatividad}

Para el enfoque que interesa al presente campo de estudio, está orientada al individuo creativo, de acuerdo con Gardner: 
Víctor Guillen G - Irma Flores A. • Evaluación formativa a procesos creativos...

La creatividad no debe considerarse como algo que sólo es inherente al cerebro, la mente o

la personalidad de un individuo por sí solo. Antes bien, debe pensarse que la creatividad surge de la interacción de tres nodos: el individuo con su propio perfil de capacidades y valores; los ámbitos para estudiar y dominar algo que existen en una cultura; y los juicios emitidos por el campo que se considera como competente dentro de una cultura (p.9).

La creatividad, en palabras de Saturnino de la Torre, se hace capacidad en la persona, estímulo en el medio, secuencia en el proceso y valor en el producto.

\section{Metodología}

Se hace necesaria la elección de un paradigma, entendido como una determinada manera de concebir e interpretar la realidad, compartida por un grupo de personas para llegar al conocimiento o producto deseado (Sandín-Esteban, 2003) y, para el caso de explorar e interpretar los sistemas de evaluación en talleres de artes visuales, se ha de seguir el rigor del método científico de investigación apoyado en una metodología cualitativa, ya que la observación a procesos de evaluación que contemple el conocimiento teórico, la creatividad e innovación y la habilidad técnica en artes; corresponden a un análisis de cualidades y características específicas; que requiere ser abierto y flexible, permitiendo identificar actitudes, interacciones y percepciones de la realidad, en un momento y espacio específicos. Como parte de tal fundamentación metodológica, la propuesta es partir desde el análisis del método de la Teoría Fundamentada (TF); el término que se refiere a la TF, Glaser la comprende como una teorización fundamentada en el análisis y unida a la recogida de datos, con un método de comparación constante. El producto de investigación final constituye una formulación teórica, o un conjunto integrado de hipótesis conceptuales, sobre el área sustantiva del objeto de estudio, para construir de tal forma una teoría que explique, para el caso presente, el fenómeno de la evaluación de procesos creativos, con base en los datos obtenidos (Giraldo-Prato, 2011, p. 80). El diseño para la observación del pilotaje se enmarcó en tres dimensiones propuestas por Alexander R. (Alexander, 2013) quien plantea la existencia de tres niveles o dimensiones de la enseñanza dentro de un aula escolar: organización, discurso y significado (ver figura 1). 


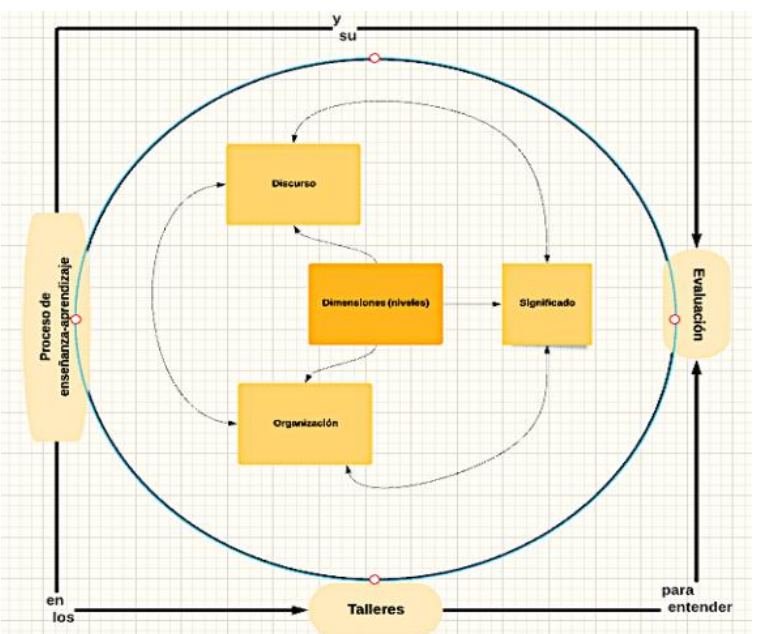

Figura 1 Dimensiones para la observación a talleres en pilotaje. Elaboración propia

Una vez finalizado del pilotaje (Artes Visuales, Universidad de Guanajuato), Se llegó a la conclusión de que la organización, el discurso y el significado, no conformaban el marco adecuado para el dimensionamiento y categorización de la evaluación al proceso creativo, por lo que en su lugar, se sustituyeron por una propuesta derivada del modelo de Flanders (1977), reinterpretada y modificada para el estudio de caso, así se consideraron algunos cambios que reflejan de manera envolvente los componentes teóricos y prácticos del fenómeno de estudio y que aporten una visión más detallada para su posterior análisis (Figura 2).

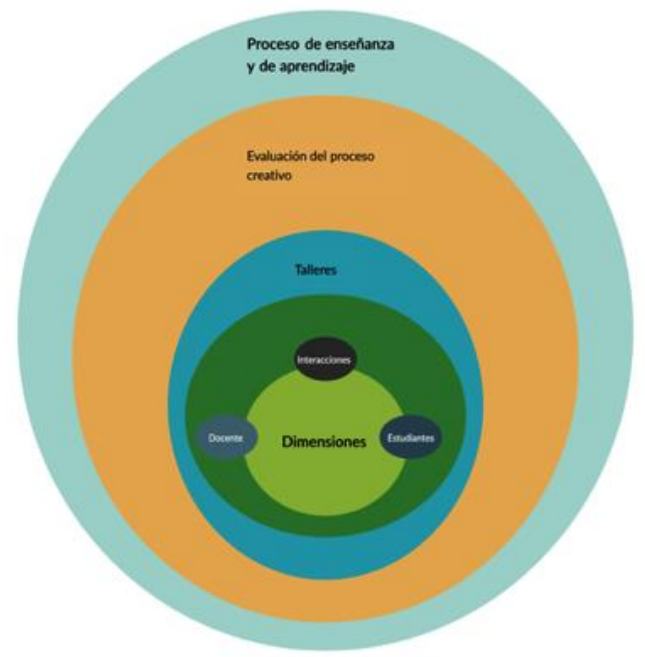

Figura 2 Teorización fundamentada. Unidad de Observación modificada, Elaborado por: Irma María Flores Alanís; Victor Alejandro Guillén García

\section{Conclusiones parciales}

- La evaluación de los procesos creativos aún está poco entendida y delimitada, por lo que cada profesor observado no tiene un solo método ni herramientas específicas, y el criterio tiende a reducirse solo a la percepción personal.

- La evaluación en talleres tiende a limitarse a parámetros y rúbricas recomendadas por el programa de estudios.

- El proceso creativo se observa y es relevante para la retroalimentación entre profesores y alumnos, pero no incide directamente en la evaluación sumativa, ya que se encuentra resistencia a evaluar aspectos creativos, por considerar que eso limita la capacidad expresiva del estudiante. 
Víctor Guillen G - Irma Flores A. • Evaluación formativa a procesos creativos...

- De acuerdo al grado o nivel del curso, los profesores perciben mayor libertad para involucrar aspectos conceptuales relacionados a la creatividad, es decir, en niveles iniciales se promueve un acompañamiento cercano para la obtención de habilidades técnicas relacionadas a manejo de materiales, con el avance en la carrera, se incrementa la valorización del discurso y línea de producción artística.

Con base en las entrevistas a expertos se contempla la existencia de una tendencia a evitar la evaluación de los procesos creativos en el sentido directo a otorgar juicios de valor respecto de los trabajos/obras artísticas de los estudiantes, ya que en su mayoría coinciden en que toda evaluación critica a los mismos, se convierte en una legitimación arbitraria y excluye a aquellos estudiantes que proponen discursos distintos a los aceptados normalmente por el gremio artístico. 


\section{Bibliografía}

Albert Gómez, M. J. (2007). La investigación educativa. Claves teóricas (1 ${ }^{\text {a }}$ edición ed.). (J. M. Cejudo, Ed.) Madrid, Madrid, España: Mc Graw Hill.

Alexander, R. (2013). Essays on pedagogy. Essays on Pedagogy, 1-212. https://doi.org/10.4324/9780203609309

Álvarez Méndez, J. M. (2001). Evaluar para conocer, examinar para excluir. Madrid, Madrid, España: Morata.

Álvarez Méndez, J. M. (2012). Pensar la evaluación como recurso de aprendizaje. En B. Jarauta Borrasca, \& F. Imbernón Muñoz, Pensando en el futuro de la educación: una nueva escuela para el siglo XXII (1 ${ }^{a}$ ed., págs. 139-158). Barcelona, Barcelona, España: Grao.

Anijovich, R. (18 de septiembre de 2017). La evaluación formativa en la enseñanza superior. Voces de la educación, 1(2), 31-38.

Barriga Monroy, M. 1. (diciembre de 2011). La investigación creación en los trabajos de pregrado y posgrado en educación artística. Recuperado el 28 de junio de 2016, de Redalyc.org: http://www.redalyc.org/articulo.oa?id=87420931021

Borgdorff, H. (2005). El debate sobre la investigación en las artes. (H. Borgdorff, Ed.) Recuperado el 28 de junio de 2016, de http://www.ahk: http://www.ahk.nl/lectoraten/onderzoek/ahkL.htm.

Borgdorff, H. (2011). The production of knowledge in artistic research. Recuperado el 1 de julio de 2016, de artun.ee: http://www.artun.ee/wp-content/uploads/2013/Borgdoff-2011.pdf Camilloni, A. R., Celman, S., Litwin, E., \& Paolou de Maté, M. d. (2001). La evaluación de los aprendizajes en el debate didáctico contemporáneo ( $3^{\mathrm{a}}$ reimpresión ed.). Buenos Aires, Buenos Aires, Argentina: Paidos.

Delors, J. e. (1996). La educación encierra un tesoro. (UNESCO, Ed.) Recuperado el 23 de febrero de 2017, de http://ceups.educacion.unmsm.edu.pe/proyeccion_archivos/educacionencierra-un-tesoro.pc

de Camilloni, A. R., Celman, S., Litwin, E., \& Paolou de MAté, M. d. (2001). La evaluación de los aprendizajes en el debate didáctico contemporáneo ( $3^{\text {a }}$ reimpresión ed.). Buenos Aires, Buenos Aires, Argentina: Paidos.

de Duve, T. (2009). An Ethics. Putting Aesthetic Transmission in Its Proper Place in the Art World. En S. H. Madoff, \& S. H. Madoff (Ed.), Art School. Propositions for the 21St Century (págs. 15-24). Cambridge, Massachusetts, USA: MIT Press.

De Ketele, J.-M. (1984). Observar para educar. Observación y evaluación en la práctica educativa (1 ${ }^{\text {a }}$ Traducción ed., Vol. XIV). (E. Bassedas, Trad.) Madrid, Madrid, España: VISOR LIBROS.

Díaz Alcaide, M. D. (2011). Sobre educación en artes plástica y visuales. Ser y Tiempo(25), 163-170.

Efland D., S., Freedman, K., \& Stuhr, P. (2003). La educación en el arte posmoderno. Barcelona, Barcelona, España: Paidós.

Eisner, E. W. (2004). El arte y la creación de la mente. El papel de las artes visuales en la transformación de la conciencia . (G. Sanchéz-Barberán, Trad.) Barcelona, Barcelona, España: Paidós.

Hernández-Arteaga, I., \& Alvarado-Pérez, J. C. (febrero-mayo de 2015). Creatividad e innovación: competencias genéricas o transversales en la formación profesional. Revista Virtual Universidad Católica del Norte, 135-151.

Herran, A. d. (2012). Universidad Autónoma de Madrid. (D. V. Vázquez, Ed.) Recuperado el 1 de julio de 2016, de http://www.uam.es/personal_pdi/profesorado/agustind/textos/procesoproducto.pdf

Lévi-Strauss. (1969). Las estructuras elementales del parentesco. (M. T. Cevasco, Trad.) Barcelona, Barcelona, España: Paidós. 
Víctor Guillen G - Irma Flores A. • Evaluación formativa a procesos creativos...

Moreno, T. (26 de mayo de 2012). La evaluación de competencias en educación. Sinéctica(39), 1-20.

Navarrete Tudela, A. (2014). Algunas anotaciones en torno a la enseñanza del arte en la enseñanza pública: el caso español. Castilla, La Mancha, España.

Okón, Y. (2005). Introducción. En A. Dorfsman, Y. Okón, A. Dorfsman, \& Y. Okón (Edits.), La Panadería. 1994-2002 (1 ${ }^{a}$ edición ed., págs. 7-13). México, Distrito Federal, México: Turner .

Osuna Barriga, J. G. (julio de 2012). Un viaje a ninguna parte: la investigación-creación como vehículo de validación institucional de la producción artística. Cuadernos de Música, Artes Visuales y Artes Escénicas, 5. Bogota, Cundinamarca, Colombia. Recuperado el 28 de junio de 2016, de Redalic.org: http://www.redalyc.org/articulo.oa?id=297023537001

Picado Gättgens, X. (2002). Criterios para realizar evaluaciones de calidad. Recuperado el 23 de junio de 2016, de Red de Revistas Científicas de America Latina y el Caribe, España y Portugal: http://www.redalyc.org/articulo.oa?id=15309702

Reynolds, K. C. (1997). Progressive Ideals and Experimental Higer Education: The Example of John Dewey and Black Mountain College. Education and Culture, 14(1). Utah, USA.

Storr, R. (2009). Dear Colleague. En S. Madoff, \& Madoff (Ed.), Art school (propositions for the 21st century) (págs. 59-60). Cambridge, Massachusetts, USA: MIT Press.

Recibido: 26 de Diciembre de 2019

Aceptado: 31 de Diciembre de 2019 